\title{
STUDY AND ANALYSIS OF ROUTING PROTOCOLS IN MOBILE AD-HOC NETWORK
}

\author{
${ }^{1}$ Uma Mani, ${ }^{2}$ Ramasamy Chandrasekaran and ${ }^{3}$ V.R. Sarma Dhulipala \\ ${ }^{1}$ Department of Computer Science and Engineering, \\ Pavendar Bharathidasan College of Engineering and Technology, Tiruchirappalli, Tamil Nadu, India \\ ${ }^{2}$ Department of Computer Science and Engineering, Annamalai University, Chidambaram, Tamil Nadu, India \\ ${ }^{3}$ Department of Physics, Anna University, \\ Bharathidasan Institute of Technology Campus, Tiruchirappalli, Tamil Nadu, India
}

Received 2013-07-19, Revised 2013-09-19; Accepted 2013-09-30

\begin{abstract}
Mobile Ad-hoc Networks (MANETs) is a network without a fixed Infrastructure. In wireless networks users expect to get access to the network security and to exchange information anytime and anywhere. The ad hoc wireless network consists of mobile networks which creates architecture for communication without the help of traditional fixed-position routers. Establishing strong routes and providing security are the main tasks in MANETs. Routing is the critical issue in the MANET and focus based on routing protocols such as Ad hoc On-demand Distance Routing Protocol (AODV), Dynamic Source Routing (DSR), Temporally Ordered Routing Algorithm (TORA) and Optimized Link State Routing (OLSR) are interesting due to their applications. OPNET is used to establish the simulations of these routing protocols the performance of the routing protocols is studied by selecting metrics. Metrics are used to select the best path for exchanging information from source to destination. Result show that AODV and TORA is suitable for larger networks while changing network topology with selected metrics.
\end{abstract}

Keywords: MANET, AODV, DSR, TORA, OLSR, WMN

\section{INTRODUCTION}

The ad-hoc network is a collection of wireless mobile nodes forming a temporary network without centralized administration (Gupta et al., 2010). Mobile Ad-hoc networks are self-organizing and self-configuring multihop wireless networks where, the structure of the network changes dynamically, due to the mobility of the nodes (Aarthy et al., 2011). Nodes in these networks utilize the same random access wireless channel and multihop forwarding. The nodes in the network not only act as hosts but also as routers that transfers data to/from other nodes in network (Patil and Sidnal, 2013).

In wireless network, security has the important feature to guarantee the protection and wired environment too in wired network. Nowadays Mobile Ad-hoc Networks (MANETs) have received appalling attention because awareness due to the automatic maintenance adequacies (Luo et al., 2002). open network architecture includes the characterstics of shared wireless medium, stringent resource constraints and highly dynamic network topology therefore, the existing security for wired network dies not support directly to the Manet thus MANET enable the following characterstics, such as anonymity and availability, to mobile users.

Ubiqutous devices incorporate some fundamental characterstics of MANET routing protocols. May be in future MANET will resembles wireless senosor network and plug and play devices. in wireless network routing is highly restrictive due to the characterstics of mobility nodes, thus protocols for routing have been getting alterted to reduce the complex of routing to move the information from source to destination. In order to get the efficient routing the protocol may take the several Corresponding Author: Uma Mani, Department of Computer Science and Engineering, Pavendar Bharathidasan College of Engineering and Technology, Tiruchirappalli, Tamil Nadu, India 
parameters to route the packets from source to destination, parameters that thake standard dimension that can be through put, wich is used by the routing protocol decides the route to transmit packet from source to it's destination. Routing algorithm initialize maintains routing tables, which contains routing information for the packets to reach its destination there is some unique way of handling the route among all protocols. There are many simulations are done based on routing protocols. We have briefly describe AODV, DSR, TORA and OLSR and finally conclude which protocol is suitable under performance metrics.

\subsection{Related Work}

Routing in MANET is difficult because, the nodes moves dynamically and there is change in network topology and requires flexible mechanism to search the routes. Due to mobile nodes and change in topology route maintain is difficult. To overcome these routing protocols use several parameters and deals with energy consumption, high error rates. Developing routing protocols for MANETs is a broad research area and many efficient routing protocols have been proposed. routing protocols are catogeries into three divisions: proactive, reactive, hybrid, these protocols gives the fundamental ways to establish the routing terms of scalability, mobility support and energy consumption (Singh et al., 2012). for correctness of routing state, the pro active approach has been used, where as reactive approach protect packet forwarding options.

The proposed work establishes the clear view of routing protocol for different parameters (delay, network load and throughput. AODV is used in highly dynamic situations and in larger network of data transmission) with the simulation tool with ns2. It provides loop free routes and repair failures or link breakages. DSR reduces control overhead, energy consumption because there is periodic routing updates. DSR repair link failures by the use of MAC layer. TORA is used for multihop, highly dynamic changes and it maintains multiple routes so there is no reaction when network topology changes. OLSR is proactive it maintains routing table for each node and there is route discovery delay for finding new routes.

In dense population of nodes the mobile TORA will be the suitable one to handle the large mobile networks, it supports for multiple routes and multicasting (Taneja et al., 2010). Related works exposes that DSR is working better than AODV, sometimes cbr traffic sources leading to the same destination (Jat and Patel, 2012) the simulation was done in qualnet simulator. The previous work done in qualnet simulator, from that results AODV shows the best performance in low density, whre as in high density both oslr and DSR performes well, oslr is good in both low and high node density (Jat and Patel, 2012) that OLSR is best suited in traffic application. for example streamin trffic, voice and video traffic. where as Implimentation in application becames traffic delay in critical factor. In this study we observed and analyzing the number of nodes by increasing and decreasing for AODV, DSR, OLSR and TORA in wireless ad-hoc network. These four routing protocols are taken because some authors wrote that DSR is best in (Kant and Kumar, 2012) AODV is best in reference (Gupta et al., 2010) TORA is best. Authors have mostly taken AODV and DSR or DSR and TORA and analyzed which routing protocol is suitable for wireless. Reactive or proactive routing enables the establishment and maintenance and mimization of communication transparency and AODV is suitable for wireless transmission (for wireless transmission AODV is suitable because it maintains a routing table so that the head of data packet need not to carry the full routing information and increases efficiency of the system. where as the full information of routing has been maintain in DSR. OLSR routing protocol is a proactive routing protocol and it can perform well with variable traffic pattern.

\subsection{Routing Protocols}

Concept of routing is to moving information from a source to a destination in an internetwork. Routing information from source to destination in an internetwork, routing involves two stages, first to form a route paths to destination and transfer the packets to destination. Routing protocols make use of several metrics to manipulate the best path for efficient routing (Malik et al., 2011).

Routing protocols for mobile ad hoc networks have different features. Regarding the way to exchange routing information, the main difference is between reactive and proactive routing protocols (Bhat et al., 2011). Static and dynamic routing are the two types of routing. Static routing remains statically in the router. If new router is added in static routing, administrator makes the changes in routing table and it does not depend on destination is active or not. Dynamic routing depends on destination network and no need of administrator it simply floods the information of added or removed nodes.

\subsection{Reactive Routing Protocols}

A reactive (or on-demand) routing protocol determines routes only when there is any packets to send. 
Ondemand routing protocol establish the routes when any packets to send, source node take care of the route when the route is not known/broken. It floods the route request packet to the networks to find the path, this lead to network clogging. Reactive routing protocols reduce overhead and it can be used in resource limited environment. On-Demand routing protocols contains low overhead compare to table driven routing protocols. In reactive routing protocols describe in detail about AODV, DSR and TORA protocol. AODV is highly dynamic so it can be used vehicular ad hoc networks and it can be used for both unicast and multicast.

\subsection{Proactive Routing Protocols}

A proactive (Table-driven) routing protocol maintain routes to all destinations of all time, regardless of whether they are needed. It maintains correct route by sending control message due to these messages waste of bandwidth if no packets to sent. Each node in the protocol maintains routing information so it is not suitable for larger networks. There is slow reaction when any link breakage or failures. The major advantage of the routing it quickly obtain routing information and establish routes. Example of proactive routing protocol is OLSR.

\subsection{Ad hoc On-Demand Distance Vector Routing Protocol (AODV)}

Ad hoc on demand distance vector routing algorithm facilitates mulithop, dynamic routing more over mobile nodes has participating between self-start inorder to establish an ad hoc network when no information is need to be passed no route is established. AODV allows mobilty nodes to respond to breakages in link and changes in network topology. AODV avoids bellmanford counting to infinity problem more over it has been designed in such a way that mobility nodes to respond even when breakages among node connecting links and also tolerate any changes in network topologythe nodes not allow to pass the lost link (Taneja and Kush, 2010). for each route entry AODV in corporates destination sequence number, by using destination sequenc number the routing can avoid loop free condition, more over the destination sequence number generated in destinatioin node, in order to obtain the route information, for requesting nodes, the sequence number has tied with the route information. If the routing is possible in two different ways the highest sequence nuber should choose a destination, requesting node is required to select the one with the highest sequence number.
The AODV has some predefiened set of identification such as (RREQ, RREP, RERR) for request,reply and error respectively. When a route to a fresh destination is required, a node broadcasts the RREQ message to find a route. A route is found when the RREQ reaches the destination itself. Suppose if a node needs to reach the destination the node needs to broadcast the RREQ message to find a route to reach the destination. when its success RREQ reach the specified destination. Unicasting enables the available route, the RREP message (reply) sends back to the destination by default for all nodes receives the RREQ message to find the destination if destination found RREP message can able to send the to the source node, the active nodes of the link has been continuously monitered in order to find the link breakage if any link break found the rrer message is to be sent in order to find the affected nodes about the loss of link. The purpose of RRER message is to indicate which destinations are now unreachable because of the link breakage.

\subsection{Dynamic Source Routing (DSR)}

Dynamic sourece routing protocol is a reactive routing protocol and DSR is called as on demand routing protocol. It is a source routing protocol and it eliminates the periodic table-update messages required in Table 1 driven approach. It is efficient and simple protocol and it can be used in multi hop wireless ad hoc networks. DSR can be used ad hoc network with mulit hop. The uniquness of DSR is self confuring and self organizing routing protocol. There are two mechanisms that DSR in corporate that is route discovery and route maintenance (Lavanya and Jeyakumar, 2011). The DSR regularly updates its route cache for new available routes. If some new available routes were found the node will directs the packet to that route. The packet has to know about the route direction. So the information about the route was set in the packet to reach its destination from its sender. DSR has two basic mechanisms for its operation:

- Route discovery

- Route maintenance

Route discovery has two messages Route Request (RREQ) and Route Reply (RREP). The basic approach is, when a node needs to send a message to a specific destination, it broadcasts the RREQ packet in the network. When RREQ found a destination, the destination node reply the packet to the route node. 
Table 1. Simulation parameters

\begin{tabular}{ll}
\hline Routing Protocols & AODV, DSR, TORA, OLSR \\
\hline Number of Wireless nodes & $5,10,15,20$ \\
Simulator & OPNET \\
Transmit Power & $0.05($ watt $)$ \\
Data rate & $11 \mathrm{Mbps}$ \\
Simulation seconds & $300(\mathrm{Sec})$ \\
\hline
\end{tabular}

\subsection{Temporally Ordered Routing Algorithm (TORA)}

The temporally ordered routing algorithm uses link reversal algorith which is comes under source initated on-demand routing protocol TORA is a distributed routing algorithm and highly adptive, efficient. TORA has three function namely creating routes, mentioning routes and erasing route (Gupta et al., 2010). TORA enables the route whenever required, not all the time. The route establishment done by directed acyclic graph, which uses the qurey update mechanism. To disclose the route establishment TORA uses Clear (CLR) packet throughout the netowkr, route establishment is done only when there is a need to communicate, it sends Query (QRY) packet to route the required flag. A qry packet contains the destination id of the node that to be communicate. The reply to a query is called an update UPD packet.

TORA is offering following characteristics:

- Distributed execution

- Loop-free routing

- Multipath routing

- Reactive or proactive route establishment and maintenance

- Minimization of communication overhead via localization of algorithmic reaction to topological changes

\subsection{Optimized Link State Routing (OLSR)}

OSLR is part of proactive link state routing, it provides the efficient link state routing for mobile ad hoc network. OSLR maintains the routing table by scater the link state, called MultiPoint Relaying (MPR). By only using MPRs to flood topology information, OLSR removes some of the redundancy of the flooding process, which may be a problem in networks with moderate to large packet loss rates. The Optimized Link State Routing (OLSR) protocol satisys the requirements of MANET.

OSLR creates two types of control: hello packets and tc packets:
- HELLO packets

- TC packets

\subsection{Simulation and Analysis}

In this study, the simulation software is optimized Network Evaluation Tool (OPNET), which is to simulate and analyze for network communication and distributed system (Sethi and Hnatyshin, 2012; Guo et al., 2011). It provides three-tier model, where the top layer is the network layer that reflects the topology of the network, the middle layer is the node layer that is composed of the corresponding protocol modules and the bottom layer is the process model that is described by finite state machines.

Aim is to analyze the performance of the routing protocols of AODV, DSR, OLSR and TORA which takes the parameters of throughput (bits per second), delay $(\mathrm{sec})$. the analyzation of routing protocols is done by the simulation tool OPNET. The communication over the wireless nodes with the transmission range of 1500 with transmission power of $0.05 \mathrm{w}$. The traffic generator starting time has set to $10 \mathrm{sec}$. The MANET traffic generator starting time was Constant $10(\mathrm{Sec})$, pause time (0) size and the duration period Constant 10 (Sec). The random mobility model was used. The simulation was 300 seconds in length.

These routing protocols can be configured on:

- MANET Stations

- WLAN workstations and servers

- Routers

\section{Performance indexes:}

- Throughput

- Delay

- Load

In the OPNET simulator first create a design of the network and by applying individual statistics to each node the performance is viewed by running DES. Then the result is viewed by view result. Figure 1 shows the workflow of OPNET simulator.

\subsection{Parameter Analysis}

There are various kinds of performance evaluation are there with respect to the parameters of the routing protocol. In this study there are three kinds of parameters are used to analyze the overall network performance, the parameters are delayed, network and throughput. 


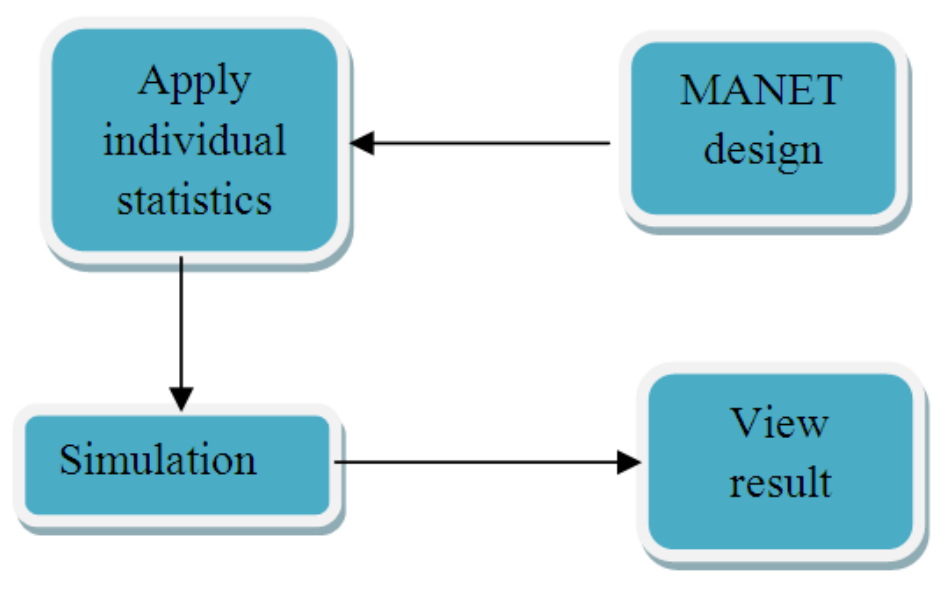

Fig. 1. Simulation flow

\subsection{Network Load}

The network load has represent with respect to bits/sec, which is put forward to wireless lan layers, by all higher layers in all wlan nodes of the network. The following graph shows the average load by differing numbers of nodes by 5,10,15 and 20. Average load is high for DSR when compared to other routing protocols. While comparing four routing protocols TORA is best suitable in larger network size due to load is less while increasing the number of nodes. Comparing TORA with AODV, TORA is best suitable in larger networks.

\subsection{End to End Delay}

End to End delay is time of packet that traverse from source to destination. Figure 3 states that The end to end delay of the data packets that are successfully received by WLAN MAC and forwarded to the higher layers. The performance routing protocol is better when a packet end-to-end delay is low. The following graphs show end to end delay provided by the four different protocols by differing numbers of nodes by 5, 10, 15 and 20. Average end to end delay is very low at 20 nodes in AODV and TORA compare to other routing protocols. End to End delay increases when the node increases. In previous OPNET papers AODV, DSR comparison made by throughput and delay in wireless mesh network. Reactive and proactive routing protocols are taken in MANET, delay and load of DSR are increased and it is not suitable for wireless transmission and this is the reason, DSR is not appropriate for wireless transmissioin, because DSR contains entire routing information so that data packet is large. hence AODV is better, the reason is that need not carry the full routing information and increases efficiency of the system. TORA is suitable for its overload is less compare to DSR. From this AODV and TORA are suitable for wireless transmission as in Fig. 2.

Mathematically end to end delay can be shown as Equation (1):

$\mathrm{D}_{\text {end-end }}=\mathrm{N}\left[\mathrm{D}_{\text {Trans }}+\mathrm{D}_{\text {prop }}+\mathrm{D}_{\text {proc }}\right]$

Where:

$\mathrm{D}_{\text {end-end }}=$ End-to-End Delay

$\mathrm{D}_{\text {trans }}=$ Transmission Delay

$\mathrm{D}_{\text {prop }}=$ Propagation Delay

$\mathrm{D}_{\text {proc }} \quad=$ Processing Delay

\subsection{Throughput}

The calculation of the through put can be manipulated as when the data reaches the destination from the source,the total data traffic in bits/sec is received and forwared it to the higher layer by the wlan MAC. Figure 4 shows the throughput performance of four routing protocols by differing numbers of nodes. Average throughput is high at the DSR protocol compares to other routing protocol. The network throughput becomes low with the decrease of the node number. the network through put is low when the number of nodes decreases the reason is that decreasing of node leads to reducing of data packets Throughput can be mathematically be defined by following Equation (2):

Throughput $($ bits $/ \mathrm{sec})=\frac{\text { Number of Delivered packets } \times \text { packet size } \times 8}{\text { Total simulation period }}$ 


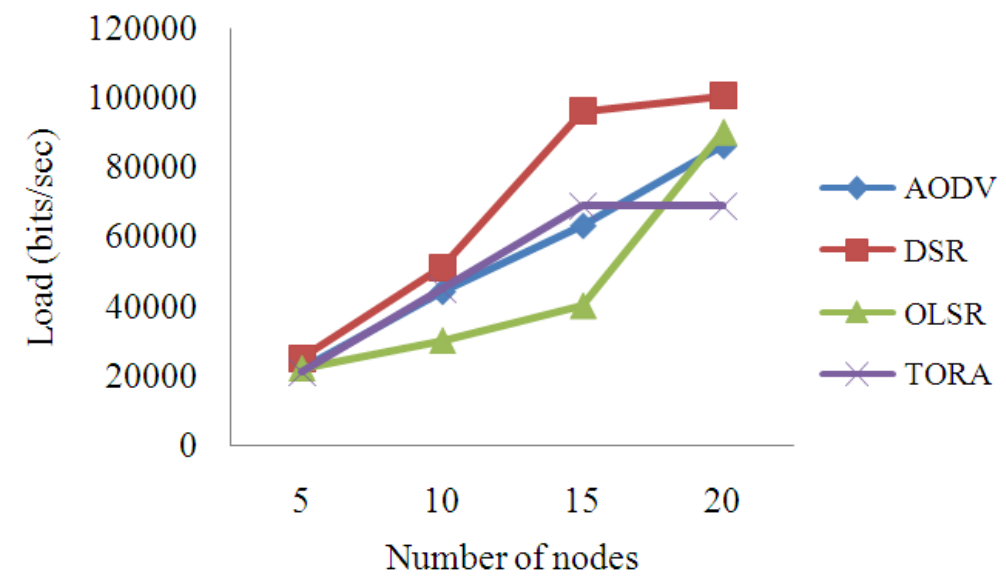

Fig. 2. Comparison of load in AODV, DSR, TORA, OLSR by increasing nodes

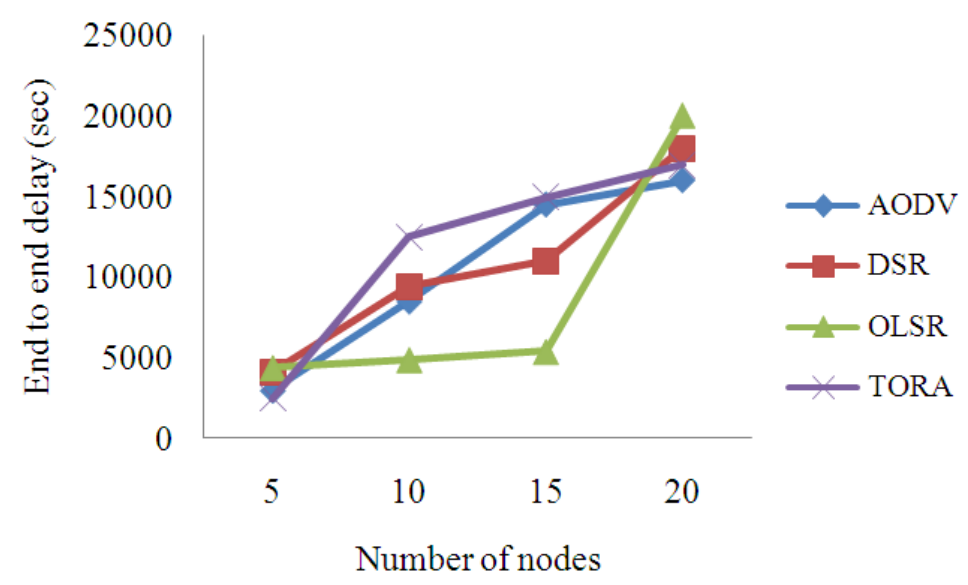

Fig. 3. Comparison of End to End Delay in AODV, DSR, TORA, OLSR by increasing nodes

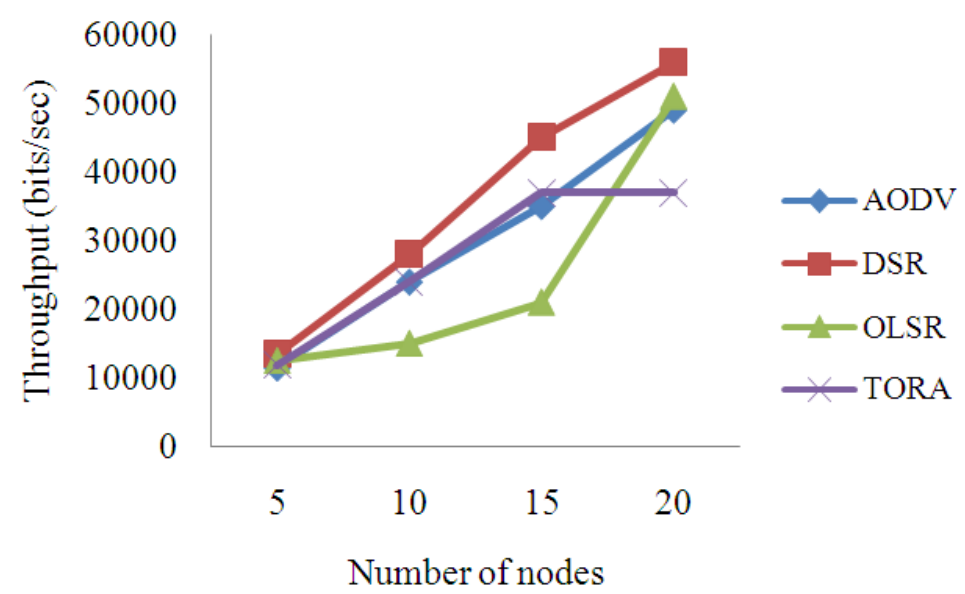

Fig. 4. Comparison of Throughput in AODV, DSR, TORA, OLSR by increasing nodes 


\section{CONCLUSION}

In this study, we addressed the reactive and proactive routing protocols by focusing on DSR, AODV, TORA and OLSR routing protocol in MANET through OPNET simulator and the results are compared. While comparing four routing protocol load, delay is low in OLSR when the number of nodes is less, so we can use OLSR in small network size. Performance is enhanced when the number of nodes is less. Throughput is high in DSR when the number of nodes increases and hence it can be used in large network size but it is not suitable for wireless transmission. TORA and AODV perform better than DSR and it is suitable in larger network size. The studies on these four routing protocols and more routing protocols in MANET will supply references to the routing protocols to design highly efficient routing protocols in WMNs in the future.

\section{REFERENCES}

Aarthy, M.P.A., M. Shanmugaraj and V.S. Dhulipala, 2011. Energy consumption analysis of multicast routing protocols in wireless ad hoc network environment. Comput. Networks Inform. Technol., 142: 412-414. DOI: 10.1007/978-3-642-19542-6_77

Bhat, M.S., D. Shwetha and J.T. Devaraju, 2011. A performance study of proactive, reactive and hybrid routing protocols using qualnet simulator. Int. J. Comput. Applic., 28: 10-17.

Guo, L., Y. Peng, X. Wang, D. Jiang and Y. Yu, 2011. Performance evaluation for on-demand routing protocols based on OPNET modules in wireless mesh networks. Comput. Electr. Eng., 37: 106-114. DOI: 10.1016/j.compeleceng.2010.10.002

Gupta, A.K., H. Sadawarti and A.K. Verma, 2010. Performance analysis of AODV, DSR and TORA routing protocols. IACSIT Int. J. Eng. Technol., 2: 226-231.
Jat, S. and B. Patel, 2012. Evaluation of Mobile nodes over different density models using MANET routing protocols. Int. J. Comput. Sci. Inform. Technol., 3: 4161-4165.

Kant, S. and K. Kumar, 2012. Performance analysis of dynamic source routing protocol in wireless mobile ad hoc network. Int. J. Eng. Res. Technol., 1: 1-7.

Lavanya, G. and A.E. Jeyakumar, 2011. An enhanced secured dynamic source routing protocol for MANETs. Int. J. Soft Comput. Eng., 10: 135-140.

Luo, H., P. Zerfos, J. Kong, S. Lu and L. Zhang, 2002. Self-securing ad hoc wireless networks. Proceedings of the 7th International Symposium on Computers and Communications, Jul. 1-4, IEEE Xplore Press, Taormina-Giardini Naxos, Italy, pp: 567-574. DOI: 10.1109/ISCC.2002.1021731

Malik, A., S. Rastogi and S. Kumar, 2011. Performance analysis of routing protocol in mobile ad hoc network using NS-2. MIT Int. J. Comput. Sci. Inform. Technol., 1: 47-50.

Patil, J.A. and N. Sidnal, 2013. Survey-secure routing protocols of MANET. Int. J. Applied Inform. Syst., 5: 8-15. 10.5120/ijais12-450875

Sethi, A.S. and V.Y. Hnatyshin, 2012. The Practical OPNET User Guide for Computer Network Simulation. 1st Edn., CRC Press, ISBN-10: 1439812055, pp: 503.

Singh, T.P., S. Dua and V. Das, 2012. Energy-efficient routing protocols in mobile ad-hoc networks. Int. J. Adv. Res. Comput. Sci. Software Eng., 2: 1-7.

Taneja, S. and A. Kush, 2010. A survey of routing protocols in mobile ad hoc networks. Int. J. Innov. Manage. Technol., 1: 279-285.

Taneja, S., A. Kush and A. Makkar, 2010. Experimental analysis of DSR, AODV using speed and pause time. Int. J. Innov. Manage., 1: 453-458. 